\title{
Letter to the editor: Theileria in loggerhead sea turtles Caretta caretta
}

\author{
Nicole I. Stacy ${ }^{1}$ (D) - Ellis C. Greiner ${ }^{2} \cdot$ Elliott R. Jacobson ${ }^{3} \cdot$ John W. Harvey ${ }^{4}$
}

Received: 2 June 2015 / Accepted: 18 June 2015 / Published online: 1 July 2015

(C) Springer-Verlag London 2015

The article by Di Santi et al. (2012) titled "Hemoparasitization by Theileria in the loggerheads Caretta caretta of the Mediterranean Sea" describes various stages of what the authors interpret as Theileria organism within erythrocytes and leukocytes of loggerhead sea turtles based on morphologic features of the suspected organism. The identification of an organism that has not been previously documented in reptiles requires its ultrastructural and molecular characterization. For example, Paparini et al. (2012) describe novel Babesia and Theileria genotypes in erythrocytes of marsupials having $0.8-2 \mu \mathrm{m}$ in diameter intra-erythrocytic inclusions with clearly visible signet ring or dot-like forms containing dark purple nuclear material consistent with a piroplasm. Molecular analysis was used for genotype confirmation and phylogenetic analysis. For reasons provided below, we do not believe that Theileria, or any infectious organism, is present in the images presented in Di Santi et al. (2012).

Nicole I. Stacy

stacyn@ufl.edu

1 Department of Large Animal Clinical Sciences, College of Veterinary Medicine, University of Florida, 2015 SW 16th Ave, PO Box 100136, Gainesville, FL 32610-0136, USA

2 Department of Infectious Disease and Pathology, College of Veterinary Medicine, University of Florida, Gainesville, FL, USA

3 Department of Small Animal Clinical Sciences, College of Veterinary Medicine, University of Florida, Gainesville, FL, USA

4 Department of Physiological Sciences, College of Veterinary Medicine, University of Florida, Gainesville, FL, USA
Figure 1c-f by Di Santi et al. (2012) describes schizonts in lymphocytes. However, we believe these to be granules within granulocytes; Fig. 1g is consistent with features of a heterophil with drying or fixation artifact rather than a Theileria schizont. Likewise, inclusions in cells in Fig. $1 \mathrm{~h}-\mathrm{j}$ do not have morphology consistent with schizonts. Figure $1 \mathrm{j}$ may be a lysed melanomacrophage, a non-specific finding in blood films of healthy reptiles (Strik et al. 2007).

Figures 2 and 3 by Di Santi et al. (2012) have images of erythrocytes that are reported to contain intraerythrocytic rounded, bacilliform, or crescent-shaped parasites. Inclusions were variably described as dividing merozoites, tiny bacilliform parasites, curved trophozoites, and gametocytes. These colorless, refractile, and variably sized "inclusions" are not consistent with Theileria organisms, but rather appear to be drying or fixation artifacts, which can be observed in erythrocytes and leukocytes of any species, especially in thicker areas of a blood film (Fig. 1 in this correspondence below).

Figure 4 a,b by Di Santi et al. are reported to show heterophils phagocytizing parasitized erythrocytes. The morphologic description of erythrophagia in loggerhead turtles has been recently addressed (Stacy and Harvey $2015)$ in response to another publication by this laboratory (Ruggiero et al. 2013). As stated previously, we disagree that erythrophagia is present in the images in which it is described. The erythrocytes are next to the heterophils but the cell membrane of heterophils does not encircle erythrocytes. The amorphous orange discoloration of the background is consistent with an artifact that may originate from drying, staining (residual stain), or smearing (cytoplasmic contents leaked from damaged heterophils). 


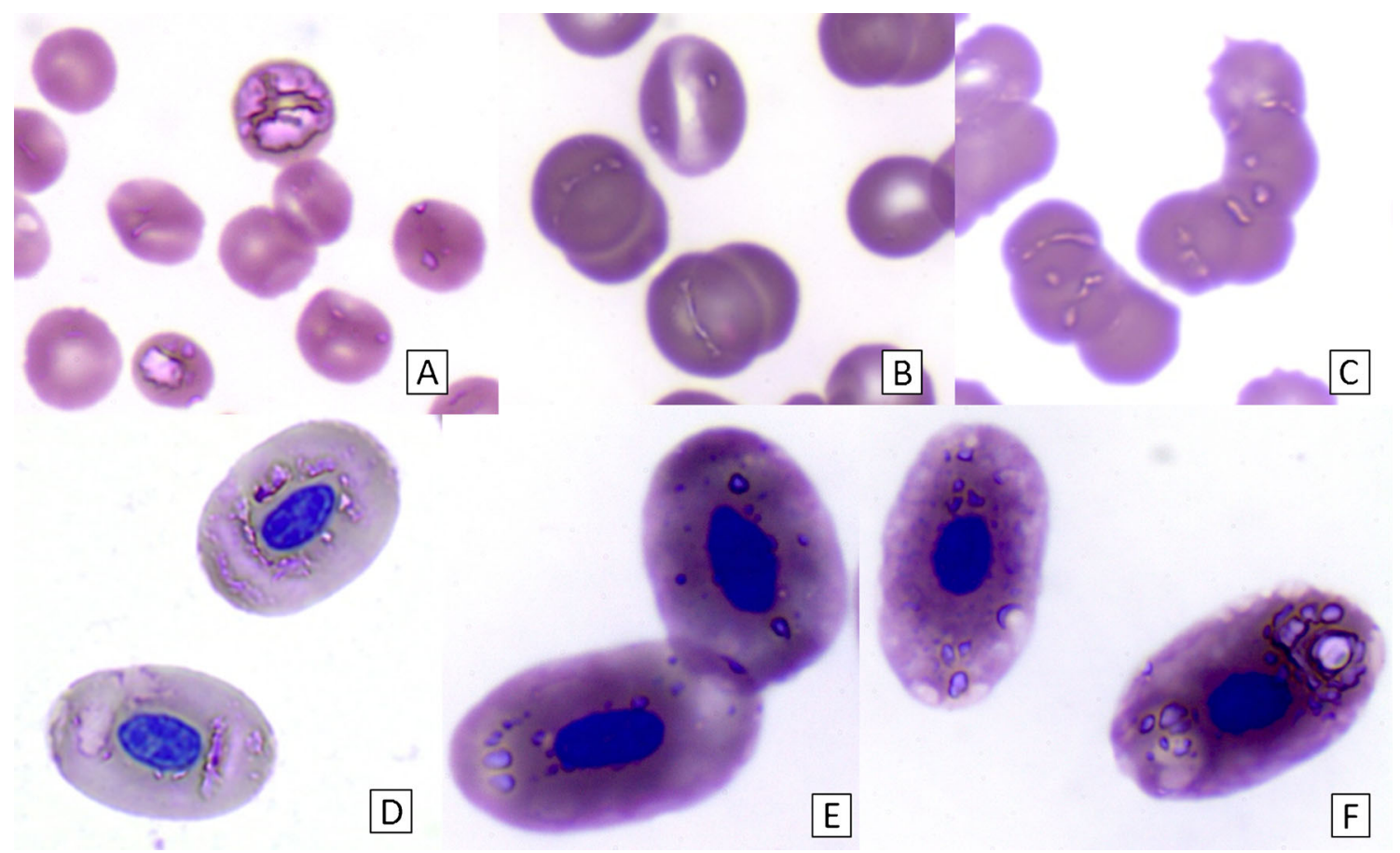

Fig. 1 Colorless, refractile, and variably sized drying or fixation artifacts in erythrocytes of a a cow, $\mathbf{b}$ sea lion, $\mathbf{c}$ manatee, $\mathbf{d}$ gopher tortoise, $\mathbf{e}$ green turtle, f Kemp's ridley sea turtle. Wright-Giemsa. $\times 100$

\section{References}

Di Santi A, Basile F, Ferretti L, Bentivegna F, Pica A (2012) Hemoparasitization by Theileria in the loggerheads Caretta caretta of the Mediterranean Sea. Comp Clin Pathol 21:63-71

Paparini A, Ryan UM, Warren K, McInnes LM, de Tores P, Irwin PJ (2012) Identification of novel Babesia and Theileria genotypes in the endangered marsupials, the woylie (Bettongia penicillata ogilbyi) and boodie (Bettongia lesueur). Exp Parasitol 131:25-30
Ruggiero MG, Ferretti L, Glomski C, Pica A (2013) Erythrophagocytosis in circulating blood of loggerhead turtles Caretta caretta: the pitting of Heinz bodies. J Exp Zool 321A:144-150

Stacy NI, Harvey JW (2015) Letter to the editor: Heinz bodies and erythrophagocytosis in the peripheral blood of loggerhead sea turtles. J Exp Zool A Ecol Genet Physiol. doi:10.1002/jez.1925

Strik NI, Alleman AR, Harr K (2007) Comparative reptile hematology circulating inflammatory cells. In: Jacobson ER (ed) Infectious diseases and pathology of reptiles: a color atlas and text. CRC Press, Boca Raton 